\title{
Eigenfrequencies of nodeless elastic vibrations locked in the crust of quaking neutron star
}

\author{
S.I. BASTRUKOV1, H.-K. CHANG, G.-T. CHEN \\ Department of Physics and Institute of Astronomy, National Tsing Hua University, \\ Hsinchu, 30013, Taiwan \\ bast@phys.nthu.edu.tw; hkchang@phys.nthu.edu.tw; d923317@oz.nthu.edu.tw \\ I. V. MOLODTSOVA \\ Joint Institute for Nuclear Research, 141980 Dubna, Russia, e-mail molod@theor.jinr.ru
}

\begin{abstract}
The Newtonian solid-mechanical theory of non-compressional spheroidal and torsional nodeless elastic vibrations in the homogenous crust model of a quaking neutron star is developed and applied to the modal classification of the quasi-periodic oscillations (QPOs) of X-ray luminosity in the aftermath of giant flares in SGR 1806-20 and SGR 1900+14. Particular attention is given to the low-frequency QPOs in the data for SGR 1806-20 whose physical origin has been called into question. Our calculations suggest that unspecified QPOs are due to nodeless dipole torsional and dipole spheroidal elastic shear vibrations.
\end{abstract}

Keywords: seismic vibrations of neutron stars; quasiperiodic oscillations in X-ray flare. PACS: 97.60.Jd; 97.10.Sj.

Published in Mod. Phys. Lett. A, Vol. 23, No. 7 (2008) pp. 477-486.

\section{Introduction}

This study was undertaken as a part of current extensive theoretical investigations of asteroseismology of a neutron star[1, 2, 3, 4, 5, 6] (see also references therein) that have been boosted by recent discovery of quasi-periodic oscillations (QPOs) of X-ray luminosity in the decaying flares of two magnetars[7, 8], SGR 1806-20 and SGR 1900+14, with concomitant suggestion to interpret this variability as caused by quake induced differentially rotational, torsional, oscillations. Following this suggestion the focus of most theoretical works is on computing the frequency spectra of odd-parity torsional mode of shear vibrations and less attention is paid to the even parity spheroidal elastic mode. However, from the viewpoint of modern global seismology [9, 10], the spheroidal vibrational mode in a solid star and planet has the same physical significance as the toroidal one in the sense that these two fundamental modes owe their existence to one and the same restoring force[11, 12, 13]. In this

\footnotetext{
${ }^{1}$ Joint Institute for Nuclear Research, 141980 Dubna, Russia. E-mail: bast@jinr.ru
} 
light there is a possibility that, by not considering both these modes on an equal footing, we may miss discovering certain essential novelties which are consequences of solid mechanical laws governing seismic vibrations of superdense matter of neutron stars. Adhering to this attitude and continuing our current investigations[13], we derive here spectral equations for the frequency of both spheroidal and torsional elastic nodeless vibrations in the solid crust of quaking neutron star and examine what conclusions can be drawn regarding low-frequency QPOs whose physical nature still remain unclear.

\section{Frequency of nodeless spheroidal and torsional elas- tic shear vibrations in homogeneous crust}

In this paper we follow the line of argument of the standard two-component, core-crust, model of quaking neutron star[14] in which crustal metal-like material (composed of nuclei dispersed in the sea of relativistic electrons) is treated as a highly robust to compressional distortions elastic material continuum of a uniform density $\rho$ characterized by constant value of shear modulus $\mu$. In this model it is presumed that the quake-induced non-compressional seismic vibrations driven by bulk force of pure shear elastic deformations (which are not accompanied by fluctuations in density $\delta \rho=-\rho \nabla_{k} u_{k}=0$ ) can be adequately modeled by equation of Newtonian, non-relativistic, solid mechanics

$$
\rho \ddot{u}_{i}=\nabla_{k} \sigma_{i k}, \quad \sigma_{i k}=2 \mu u_{i k}, \quad u_{i k}=\frac{1}{2}\left[\nabla_{i} u_{k}+\nabla_{k} u_{i}\right], \quad u_{k k}=\nabla_{k} u_{k}=0 .
$$

From now on $u_{i}(\mathbf{r}, t)$ stands for the field of material displacements in the crust of the depth $\Delta R=R-R_{c}$ with $R$ and $R_{c}$ being radii of star and core, respectively. The linear relation between tensors of shear elastic stresses $\sigma_{i k}$ and shear deformations or strains $u_{i k}$ is the Hooke's law of elastic (reversal) shear deformations.

In what follows we focus on poorly investigated regime of nodeless shear vibrations in which the fields of oscillating material displacement subject to the vector Laplace equation[13.

$$
\nabla^{2} \mathbf{u}(\mathbf{r}, t)=0
$$

This last equation can be thought of as the long wavelength limit of vector Helmholtz equation describing standing-wave regime of vibrations [6, 13]. In these latter works it has been shown that the eigenfrequency problem of global nodeless vibrations of a solid star can be unambiguously solved with aid of the Rayleigh's energy method. In the present paper this method is extended to the case of spheroidal nodeless vibrations which are considered in one line with torsional ones.

The stating point of the energy variational method is the integral equation of the energy balance

$$
\frac{\partial}{\partial t} \int \frac{\rho \dot{u}^{2}}{2} d \mathcal{V}=-\int \sigma_{i k} \dot{u}_{i k} d \mathcal{V}=-2 \int \mu u_{i k} \dot{u}_{i k} d \mathcal{V}
$$


which is obtained by scalar multiplication of equation of solid mechanics, (1), with $u_{i}$ and integration over the volume of seismogenic layer. The field $\mathbf{u}(\mathbf{r}, t)$ can be conveniently represented in the following separable form

$$
\mathbf{u}(\mathbf{r}, t)=\mathbf{a}(\mathbf{r}) \alpha(t)
$$

with the field of instantaneous (time-independent) displacements $\mathbf{a}(\mathbf{r})$ obeying, as follows from (2), to equations

$$
\nabla^{2} \mathbf{a}(\mathbf{r})=0, \quad \nabla \cdot \mathbf{a}(\mathbf{r})=0
$$

and $\alpha(t)$ stands for the temporal amplitude of vibrations. Inserting (44) in (3) we arrive at equation for $\alpha(t)$ having the form of standard equation of normal oscillations

$$
\begin{aligned}
& \frac{d E}{d t}=0, \quad E=\frac{M \dot{\alpha}^{2}}{2}+\frac{K \alpha^{2}}{2} \quad \rightarrow \quad \ddot{\alpha}+\omega^{2} \alpha=0, \quad \omega^{2}=\frac{K}{M}, \\
& M=\int \rho a_{i} a_{i} d \mathcal{V}, \quad K=2 \int \mu a_{i k} a_{i k} d \mathcal{V} \quad a_{i k}=\frac{1}{2}\left[\nabla_{i} a_{k}+\nabla_{k} a_{i}\right] .
\end{aligned}
$$

The solenoidal fields of instantaneous material displacements in two fundamental modes of nodeless vibrations - the spheroidal (normally abbreviated as ${ }_{0} s_{\ell}$ ) and the toroidal (abbreviated as ${ }_{0} t_{\ell}$ ), are determined by two fundamental (orthogonal and different in parity) solutions to the vector Laplace equation which are uniquely defined by the general solution to the scalar Laplace equation $\nabla^{2} \chi(\mathbf{r})=0$. In spherical coordinates with fixed polar axis, the solution of (5) corresponding to nodeless spheroidal vibrations, $\mathbf{a}_{s}$, is given by the even parity poloidal (polar) vector field and instantaneous displacements in the torsional mode, $\mathbf{a}_{t}$, are described by the odd parity toroidal (axial) vector field:

$$
\begin{aligned}
& \mathbf{a}_{s}=\nabla \times \nabla \times(\mathbf{r} \chi), \quad \mathbf{a}_{t}=\nabla \times(\mathbf{r} \chi), \\
& \chi(\mathbf{r})=f_{\ell}(\mathbf{r}) P_{\ell}(\cos \theta), \quad f_{\ell}(\mathbf{r})=\left[\mathcal{A}_{\ell} r^{\ell}+\mathcal{B}_{\ell} r^{-(\ell+1)}\right] .
\end{aligned}
$$

Henceforth $P_{\ell}(\cos \theta)$ stands for the Legendre polynomial of multipole degree $\ell$ and $\mathcal{A}_{\ell}$ and $\mathcal{B}_{\ell}$ are the arbitrary constants to be eliminated from boundary conditions on the core-crust interface and on the star surface. Thus, in order to obtain the frequency spectra of both spheroidal and torsional nodeless vibrations one need to specify first these constants and then to compute integrals for integral parameters of vibrations, that is, the inertia $M$ and the stiffness $K$. Some useful mathematical details of such calculations can be found in[13].

\subsection{Spheroidal mode}

The poloidal field of nodeless instantaneous displacement $\mathbf{a}_{s}$ in s-mode is irrotational: $\nabla \times$ $\mathbf{a}_{s}=0$. To specify $\mathcal{A}_{\ell}$ and $\mathcal{B}_{\ell}$ we adopt on the core-crust interface, $r=R_{c}$, the condition of impenetrability of seismic perturbation in the core. On the star edge, $r=R$, we impose 
the condition that the radial velocity of material displacements equals the rate of spheroidal distortions of the star surface[15]

$$
\left.u_{r}\right|_{r=R_{c}}=0,\left.\quad \dot{u}_{r}\right|_{r=R}=\dot{R}(t), \quad R(t)=R\left[1+\alpha(t) P_{\ell}(\cos \theta)\right] .
$$

The solution of resultant algebraic equations leads to following values of arbitrary constants

$$
\mathcal{A}_{\ell}=\frac{\mathcal{N}_{\ell}}{\ell(\ell+1)}, \quad \mathcal{B}_{\ell}=-\frac{\mathcal{N}_{\ell}}{\ell(\ell+1)} R_{c}^{2 \ell+1}, \quad \mathcal{N}_{\ell}=\frac{R^{\ell+3}}{R^{2 \ell+1}-R_{c}^{2 \ell+1}} .
$$

Tedious but simple calculation of integrals for inertia $M$ and stiffness $K$, given by (7), with poloidal field $\mathbf{a}_{s}$ yields

$$
\begin{aligned}
& M_{s}(\ell, \lambda)=\frac{4 \pi R^{5} \rho}{\ell(2 \ell+1)\left(1-\lambda^{2 \ell+1}\right)}\left[1+\frac{\ell}{(\ell+1)} \lambda^{2 \ell+1}\right], \\
& K_{s}(\ell, \lambda)=8 \pi R^{3} \mu \frac{(\ell-1)\left(1-\lambda^{2 \ell-1}\right)}{\ell\left(1-\lambda^{2 \ell+1}\right)^{2}}\left[1+\frac{\ell(\ell+2)}{\ell^{2}-1} \frac{\lambda^{2 \ell-1}\left(1-\lambda^{2 \ell+3}\right)}{\left(1-\lambda^{2 \ell-1}\right)}\right], \\
& \lambda=\frac{R_{c}}{R}=1-h \quad h=\frac{\Delta R}{R} .
\end{aligned}
$$

The fractional frequency of nodeless spheroidal irrotational shear vibrations as a function of multipole degree $\ell$ is given by

$$
\begin{aligned}
& \frac{\omega_{s}^{2}(\ell)}{\omega_{0}^{2}}=\frac{2(2 \ell+1)}{\left(1-\lambda^{2 \ell+1}\right)}\left[\frac{\left(\ell^{2}-1\right)\left(1-\lambda^{2 \ell-1}\right)+\ell(\ell+2) \lambda^{2 \ell-1}\left(1-\lambda^{2 \ell+3}\right)}{(\ell+1)+\ell \lambda^{2 \lambda+1}}\right], \\
& \omega_{0}=\frac{c_{t}}{R}, \quad c_{t}=\sqrt{\frac{\mu}{\rho}}, \quad\left[\omega_{0}=2 \pi \nu_{0}, \quad \omega_{s}(\ell)=2 \pi \nu\left({ }_{0} s_{\ell}\right)\right] .
\end{aligned}
$$

It worth noting that in the limit of zero-size radius of the core, $\lambda=\left(R_{c} / R\right) \rightarrow 0$, when entire volume of the star sets in vibrations, we regain the early obtained spectral formula for global nodeless spheroidal nodeless shear vibrations $[13] \nu\left({ }_{0} s_{\ell}\right)=\nu_{0}[2(2 \ell+1)(\ell-1)]^{1 / 2}$ showing that the lowest overtone of the global nodeless spheroidal oscillations in the entire volume of the star is of quadrupole degree, $\ell=2$ (see also[17]). In the meantime, the lowest overtone of spheroidal vibrations trapped in the crust is of the dipole degree, $\ell=1$. This suggests that the dipole overtone can be considered as a signature of spheroidal vibrations locked in the crust. It is remarkable that dipole vibration can be thought of as, so called, Goldstone's soft mode whose most conspicuous feature is that the frequency as a function of intrinsic parameter $\lambda$ of oscillating system $\omega_{s}(\ell=1, \lambda) \rightarrow 0$, when $\lambda \rightarrow 0$. In the model under consideration this parameter is given by $\lambda=\left(R_{c} / R\right)$. The limit $\lambda=0$ belongs to translation displacement of the center-of-mass of the star, not a vibration; this is clearly seen from the equation for energy (Hamiltonian) of harmonic oscillations (6).

In the upper panel of Fig.1 we plot the fractional frequency $\omega_{s}(\ell) / \omega_{0}$ as a function of $h=\Delta R / R$. In the next section we show how the input parameters of obtained spectral equation (15), namely, the natural unit of frequency $\nu_{0}$ and the depth $h$ of seismogenic layer can be extracted from the data on QPOs for SGS. 


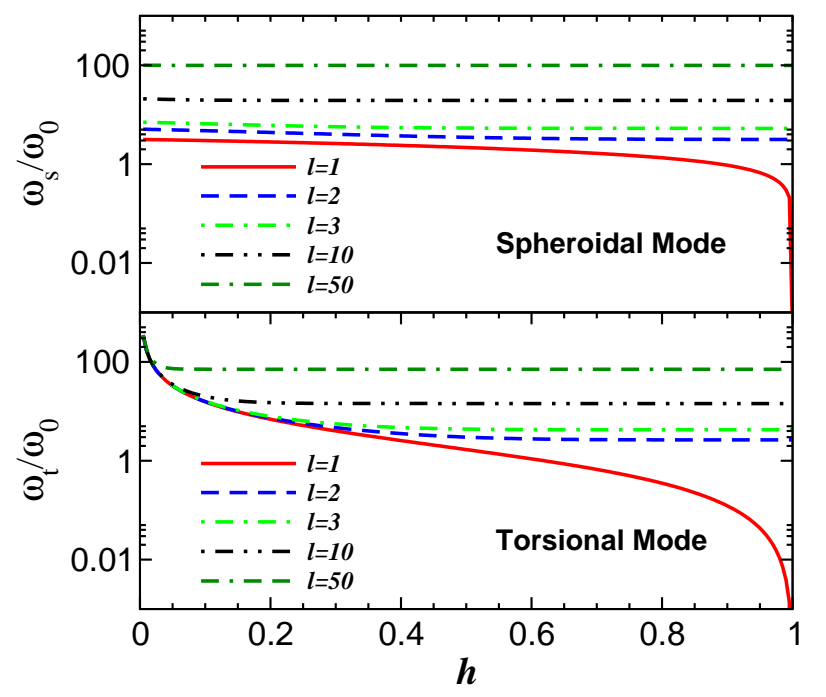

Figure 1: Fractional frequency of nodeless spheroidal and torsional elastic oscillations as a function of fractional depth of seismogenic layer.

\subsection{Torsion mode}

For the torsional oscillations locked in the crust, the constants $\mathcal{A}_{\ell}$ and $\mathcal{B}_{\ell}$ are eliminated from the following boundary conditions

$$
\begin{aligned}
& \left.u_{\phi}\right|_{r=R_{c}}=0,\left.\quad u_{\phi}\right|_{r=R}=\left[\boldsymbol{\phi}_{R} \times \mathbf{R}\right]_{\phi}, \\
& \boldsymbol{\phi}_{R}=\alpha(t) \nabla_{\hat{\mathbf{n}}} P_{\ell}(\zeta), \quad \nabla_{\hat{\mathbf{n}}}=\left(0, \frac{\partial}{\partial \theta}, \frac{1}{\sin \theta} \frac{\partial}{\partial \phi}\right) .
\end{aligned}
$$

First is the no-slip condition on the core-crust interface, $r=R_{c}$, implying that the amplitude of differentially rotational oscillations is gradually decreasing from the surface to the core. The boundary condition on the star surface, $r=R$, is dictated by symmetry of the general toroidal solution of the vector Laplace equation. The support of this last boundary condition lends further considerations showing that it leads to correct expression for the moment of inertia of a rigidly rotating star. The resultant algebraic equations steaming from above boundary conditions lead to

$$
\mathcal{A}_{\ell}=\mathcal{N}_{\ell}, \quad \mathcal{B}_{\ell}=-\mathcal{N}_{\ell} R_{c}^{2 \ell+1}, \quad \mathcal{N}_{\ell}=\frac{R^{\ell+2}}{R^{2 \ell+1}-R_{c}^{2 \ell+1}}
$$


Tedious calculation of integrals for $M_{t}$ and $K_{t}$ leads to

$$
\begin{aligned}
& M_{t}=\frac{4 \pi \ell(\ell+1)}{(2 \ell+1)(2 \ell+3)} \frac{\rho R^{5}}{\left(1-\lambda^{2 \ell+1}\right)^{2}} \times \\
& {\left[1-(2 \ell+3) \lambda^{2 \ell+1}+\frac{(2 \ell+1)^{2}}{2 \ell-1} \lambda^{2 \ell+3}-\frac{2 \ell+3}{2 \ell-1} \lambda^{2(2 \ell+1)}\right],} \\
& K_{t}=\frac{4 \pi \ell\left(\ell^{2}-1\right)}{2 \ell+1} \frac{\mu R^{3}}{\left(1-\lambda^{2 \ell+1}\right)}\left[1+\frac{(\ell+2)}{(\ell-1)} \lambda^{2 \ell+1}\right] \\
& \lambda=\frac{R_{c}}{R}=1-h \quad h=\frac{\Delta R}{R} .
\end{aligned}
$$

In the limit of zero-size radius of the core, $\lambda=\left(R_{c} / R\right) \rightarrow 0$, corresponding to torsional oscillations in the entire volume of the star we regain the early obtained spectral formula for the global nodeless torsional elastic vibrations $\nu\left({ }_{0} t_{\ell}\right)=\nu_{0}[(2 \ell+3)(2 \ell-1)]^{1 / 2}$ showing that in case of global torsional oscillations the lowest overtone is of quadrupole degree [6, 13, 17]. However, this is not the case when we consider torsional nodeless oscillations locked in the seismogening layer of finite depth $\Delta R=R-R_{c}$. For $\ell=1$, equations (20) and (21) are reduced to

$$
\begin{aligned}
& M_{t}(\ell=1, \lambda)=\frac{8 \pi \rho R^{5}}{15\left(1-\lambda^{3}\right)^{2}}\left[1-5 \lambda^{3}+9 \lambda^{5}-5 \lambda^{6}\right] \\
& K_{t}(\ell, \lambda)=8 \pi \mu R^{3} \frac{\lambda^{3}}{\left(1-\lambda^{3}\right)}, \\
& \omega_{t}^{2}(\ell=1, \lambda)=\omega_{0}^{2} \frac{15 \lambda^{3}\left(1-\lambda^{3}\right)}{(1-\lambda)^{3}\left(1+3 \lambda+6 \lambda^{2}+5 \lambda^{3}\right)} \quad 0 \leq \lambda<1 .
\end{aligned}
$$

In the limit when the core radius tends to zero, the stiffness $K_{t}(\ell=1, \lambda=0) \rightarrow 0$ and the mass parameter getting the form of the moment of inertia of absolutely rigid solid star of mass $\mathcal{M}$ and radius $R$ : $M_{t}(\ell=1, \lambda=0)=(2 / 5) \mathcal{M} R^{2}$. This latter case corresponds to the rigid rotation. The above consideration again shows that the dipole overtone exhibits features of the Goldstone soft mode owing its emergence to the trapping of torsional shear oscillations in the peripheral crust of finite depth.

The general spectral equation for the fractional frequency of nodeless torsional oscillations of arbitrary multipole degree $\ell$, computed with aid of equations (20) and (21), can be presented in the following analytic form

$$
\begin{aligned}
& \frac{\omega_{t}^{2}(\ell)}{\omega_{0}^{2}}=\frac{\nu^{2}\left({ }_{0} t_{\ell}\right)}{\nu_{0}^{2}}=[(\ell+2)(\ell-1)] p_{\ell}\left(\nu_{0}, \lambda\right) \\
& p_{\ell}\left(\nu_{0}, \lambda\right)=4\left[1-\frac{1}{2(\ell+2)}\right]\left[1+\frac{1}{2(\ell-1)}\right]\left(1-\lambda^{2 \ell+1}\right) \times \\
& \left\{1-\frac{\ell-\lambda^{2 \ell+1}\left[(\ell+2)+(2 \ell-1)(2 \ell+3)-(2 \ell+1)^{2} \lambda^{2}+(2 \ell+3) \lambda^{2 \ell+1}\right]}{(2 \ell-1)-\lambda^{2 \ell+1}\left[(2 \ell-1)(2 \ell+3)-(2 \ell+1)^{2} \lambda^{2}+(2 \ell+3) \lambda^{2 \ell+1}\right]}\right\} .
\end{aligned}
$$




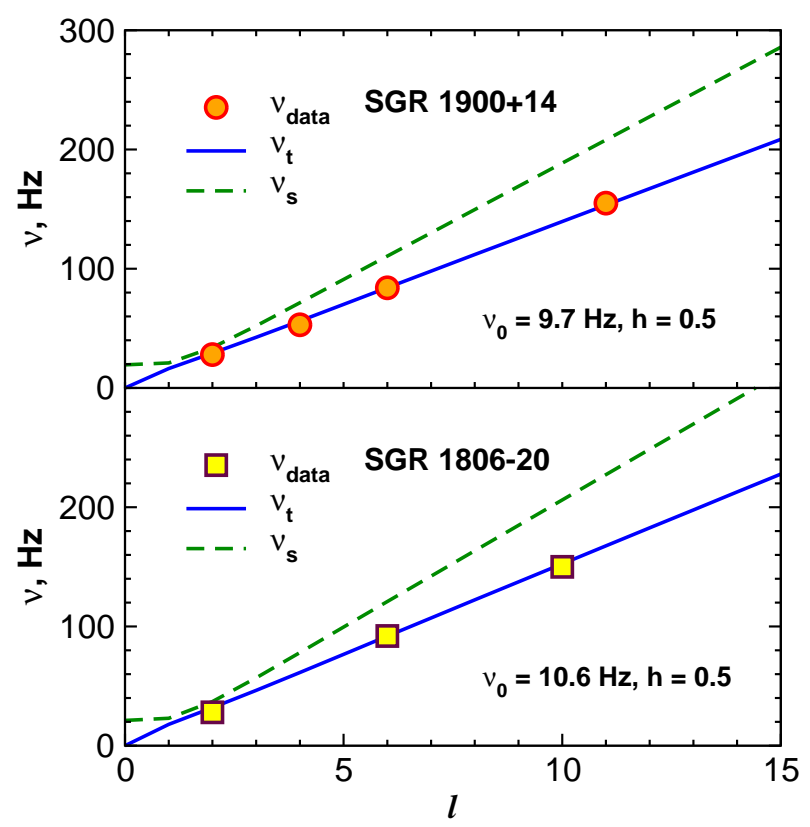

Figure 2: Theoretical curves for the frequency of spheroidal (dashed) and torsional (solid) nodeless elastic oscillations computed with aid of spectral formulae for frequency of spheroidal, eq.(15), and torsional, eqs. (26)-(27), modes as functions of multipole degree in juxtaposition with data (symbols) on QPOs for SGR 1900+14 and for SGR 1806-20.

The usefulness of such representation is extensively discussed in [6]. The fractional frequency as a function of $h=\Delta R / R$ is pictured in down panel of Fig.1 which show that the lowest overtone of torsional vibrations trapped in the crust is of dipole degree and that dipole overtone of differentially rotational vibrations of the crust against core possesses properties of the Goldstone's soft mode.

\section{Application to SGR 1900+14 and SGR 1806-20}

The obtained spectral formulae (15) and (26)-(27) describe the frequencies of both spheroidal and torsional nodeless oscillations as functions of the multipole degree $\ell$. The natural unit of frequency $\nu_{0}$ of shear elastic vibrations and the fractional depth $h$ of peripheral seismogenic layer are input parameters carrying information about material properties of neutron star matter (density, shear modulus) and geometrical sizes of star and seismoactive zone. Considering the observation data for SGR 1900+14 and SGR 1806-20, we demonstrate here how the obtained spectral equations can be used to eliminate some uncertainties in identification of QPOs.

First, we examine the agreement of obtained spectral formula (26) -(27) for torsion mode with identification of the QPOs frequencies from interval $30 \leq \nu \leq 200 \mathrm{~Hz}$ with frequencies of nodeless torsional vibrations of multipole degree $\ell$ from interval $2 \leq \ell \leq 12$ suggested 


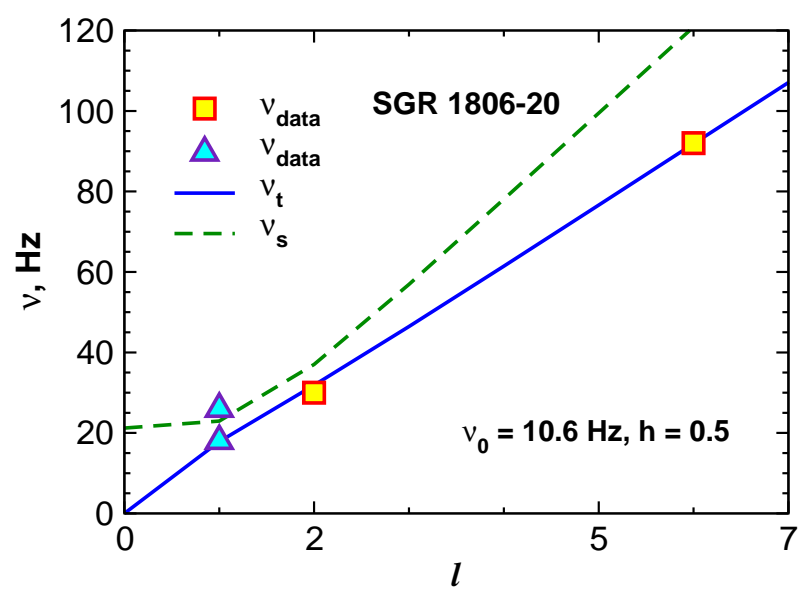

Figure 3: Theoretical predictions for the frequency of spheroidal (dashed) and torsional (solid) nodeless elastic oscillations as a function of multipole degree $\ell$ in juxtaposition with data (symbols) on QPOs during the flare of SGR 1806-20. Based on the results of this latter work, our calculations suggest that low frequency QPOs, pictured by triangles, can be identified as dipole toroidal and dipole spheroidal nodeless vibration, respectively: $\nu\left({ }_{0} t_{1}\right)=$ $18 \mathrm{~Hz}$ and $\nu\left({ }_{0} s_{1}\right)=26 \mathrm{~Hz}$.

in[3]. In so doing we use the proposed in these latter works identification of QPOs in SGR $1900+14$ data [namely, $\nu\left({ }_{0} t_{2}\right)=28 \mathrm{~Hz} ; \nu\left({ }_{0} t_{4}\right)=53 ; \mathrm{Hz} \nu\left({ }_{0} t_{6}\right)=84 \mathrm{~Hz}, \nu\left({ }_{0} t_{11}\right)=155 \mathrm{~Hz}$ borrowed from Table 1 of paper[3]] as reference points and vary parameters $\nu_{0}$ and $h$ entering our spectral formula (15) for torsional mode so as to attain the best fit of these points. The result of this procedure is shown in upper panel of Fig.2 by solid line. Then, making use of the fixed in the above manner parameters $\nu_{0}$ and $h$, we compute (with the aid of spectral formula (15) ) the frequency of spheroidal mode $\nu\left({ }_{0} s_{\ell}\right)$. The application of this procedure to modal analysis of QPOs data for SGR 1806-20 is pictured in down panel of Fig.2. Based on proposed in the above mentioned paper identification of the following points $\nu\left({ }_{0} t_{2}\right)=30$ $\mathrm{Hz} ; \nu\left({ }_{0} t_{6}\right)=92 \mathrm{~Hz}$ and $\nu\left({ }_{0} t_{10}\right)=150 \mathrm{~Hz}$ we extract parameters $\nu_{0}$ and $h$ entering in our spectral formulae for torsional mode, equations (26)-(27).

In Fig.3 set out very clearly the case for identifying two non-identified before lowfrequency QPOs in data for SGR 1806-20 (Israel et al 2005), namely $\nu=18 \mathrm{~Hz}$ and $\nu=26$ $\mathrm{Hz}$, highlighted by triangles.

\section{Summary}

The obtained spectral formulae for the frequency of nodeless elastic vibrations trapped in the finite-depth seismogenic layer may be of some interest in its own right from the viewpoint of general theoretical seismology [9] in the sense that they can be utilized in the study of seismic vibrations of more wide class of solid celestial objects such as Earth-like planets. One of 
the remarkable feature of considered model is that the dipole nodeless overtones possess properties of Goldstone soft modes, that is, the dipole overtones emerge if and on only if the elastic vibrations turn out to be locked in the peripheral layer of finite thickness. It is shown that obtained spectral equations are consistent with the existence treatment of low-frequency

QPOs in the X-ray luminosity of flares SGR 1900+14 and SGR 1806-20 as caused by quakeinduced torsional nodeless vibrations. What is newly disclosed here is that previously nonidentified low-frequency QPOs in data for SGR 1806-20 can be attributed to nodeless dipole torsional and spheroidal vibrations, namely, $\nu\left({ }_{0} t_{1}\right)=18 \mathrm{~Hz}$ and $\nu\left({ }_{0} s_{1}\right)=26 \mathrm{~Hz}$.

\section{Acknowledgements}

This work is partly supported by NSC of Taiwan, under grants NSC-096-2811-M-007-012, NSC-096-2628-M-007-012-MY3 and NSC-097-2811-M-007-03.

\section{References}

[1] A. L. Piro, Astrophys. J. 634, L153 (2005).

[2] K. Glampedakis, L. Samuelsson and N. Andersson, Mon. Notes Roy. Astron. Soc. 371 L74 (2006)

[3] L. Samuelsson L. and N. Andersson, Astrophys. and Space Sci. 308, 581 (2006).

[4] U. Lee, Mon. Notes Roy. Astron. Soc. 374, 1015 (2007).

[5] Yu. Levin, Mon. Notes Roy. Astron. Soc. 377, 159 (2007).

[6] S. I. Bastrukov, H-K. Chang, J. Takata, G-T. Chen and I. V. Molodtsova, Mon. Notes Roy. Astron. Soc. 382, 849 (2007)

[7] G. L. Israel et al, Astrophys. J. 628, L53 (2005).

[8] T. E. Strohmayer and A. L. Watts, Astrophys. J. 653, 593 (2005).

[9] T. Lay and T. C. Wallace, Modern Global Seismology (Academic Press, 1995).

[10] K. Aki and P. G. Richards, Quantitative Seismology (University Science Books, 2003).

[11] P. N. McDermott, H. M. Van Horn, C. J. Hansen, Astrophys. J. 325, 725 (1988).

[12] S. I. Bastrukov, F. Weber and D. V. Podgainy, J. Phys. G. 25, 107 (1999).

[13] S. I. Bastrukov, H.-K. Chang, Ş. Mişicu, I. V. Molodtsova and D. V. Podgainy, Int. J. Mod. Phys. A 22, 3261 (2007). 
[14] L. M. Franco, B. Link and R. I. Epstein, Astrophys. J. 543, 987 (2000).

[15] H. Lamb H., Hydrodynamics (Dover, 1945).

[16] R. P. Lapwood and T. Usami, Free Oscillations of the Earth (Cambridge University Press, 1981).

[17] S. I. Bastrukov, D. V. Podgainy, J. Yang and F. Weber, JETP 95, 789 (2002). 